\title{
Myers-Briggs Type Indicator Personality Types of Ophthalmology Residents
}

\author{
Mohamad Haidar, BA, MS IV ${ }^{1}$ Faisal Ridha, MD 2,3 John Ling, MD ${ }^{4}$ Mashal Akhter, MD ${ }^{5}$ \\ Laura Kueny, $\mathrm{MD}^{6}$ Osama Sabbagh, $\mathrm{MD}^{7}$ Chaesik Kim, $\mathrm{PhD}^{2}$ Katrina Chin Loy, $\mathrm{MD}^{8}$
}

${ }^{1}$ Department of Ophthalmology, Ohio University Heritage College of Osteopathic Medicine, Athens, Ohio

${ }^{2}$ Department of Ophthalmology, Kresge Eye Institute, Detroit, Michigan

${ }^{3}$ Wayne State University, Detroit, Michigan

${ }^{4}$ SoCalEye, Long Beach, California

${ }^{5}$ Department of Ophthalmology, University of Southern California, Los Angeles, California

${ }^{6}$ ABC Children's Eye Specialists Phoenix, Phoenix, Arizona

J Acad Ophthalmol 2021;13:e158-e162.
Address for correspondence Katrina Chin Loy, MD, Department of Ophthalmology, Howard University College of Medicine, Tower Building, 2041 Georgia Ave NW \#2100, Washington, DC 20060 (e-mail: Katrina.chinloy@howard.edu).

${ }^{7}$ Department of Ophthalmology, University of Kentucky/Retina Associates of Kentucky, Lexington, Kentucky

${ }^{8}$ Department of Ophthalmology, Howard University College of Medicine, Washington, District of Columbia

\begin{abstract}
Keywords

- resident

- applicant

- training program

- fellowship

- personality

- personality inventory

- assessment tools

- holistic evaluation
\end{abstract}

Objective This study attempts to use the Myers-Briggs Type Indicator (MBTI) to analyze personality types among current and recent ophthalmology residents. We aimed to evaluate the prevalence rates of each specific personality type in ophthalmology, and whether these changed by level of training, training program, or fellowship selection. The study aimed to evaluate whether certain personality types are more prevalent in ophthalmology as a unique medical specialty. This can help understand specialty choice and potentially predict trends in specialty selection.

Study Design After obtaining institutional review board approval from Howard University Hospital, an electronic version of the MBTI questionnaire, form M, was sent to participants. In addition to the questionnaire, participants responded to four questions inquiring about home program, postgraduate training level, subspecialty interest, and work environment (if applicable). The anonymous responses of the surveys were automatically scored on google forms, and the results were analyzed by using StatView statistical analysis.

Setting This study was conducted at Howard University, Georgetown University, George Washington University, University of Texas Medical Branch at Galveston, and Kresge Eye Institute.

Participants A total of 66 current residents and recent graduates of five residency programs were involved in this study.

Main Outcomes and Measures This study evaluated four-letter personality type from each participant.

Results Ophthalmology residents were statistically more likely to be identified in the categories of extroversion (E) than introversion (I) $(p=0.049)$, thinking $(T)$ than feeling (F) $(p=0.027)$, and judging (J) than perceiving (P) $(p=0.007)$, with no statistically significant difference between sensing (S) and intuition (N). ENTP, EST], and IST] were received

February 16, 2021 accepted after revision April 26, 2021
DOI https://doi.org/ $10.1055 / \mathrm{s}-0041-1732346$ ISSN 2475-4757.

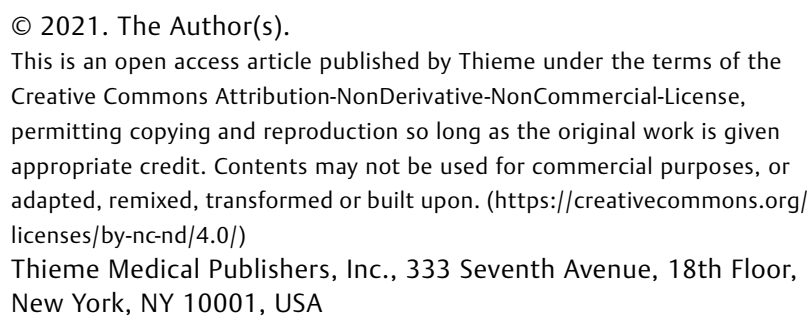
appropriate credit. Contents may not be used for commercial purposes, or adapted, remixed, transformed or built upon. (https://creativecommons.org/ licenses/by-nc-nd/4.0/) Thieme Medical Publishers, Inc., 333 Seventh Avenue, 18th Floor, New York, NY 10001, USA 
the most common personality types, each comprising $13.6 \%$ of the sample population. The ratio of $\mathrm{J}: \mathrm{P}$ was found to increase as training level increased, beginning with postgraduate 2 nd year until graduate level.

Conclusion Certain personality types are more common among ophthalmology residents in our cohort from five different training programs. It is possible that individual types change over the course of residency training and career. Understanding that these findings exist can be used as a baseline for future research in terms of potential predictors for applicants, of resident knowledge base, and personality changes over the course of one's training.

The Myers-Briggs Type Indicator (MBTI), based on Carl Jung's theory of psychological type preferences, has been the most widely used assessment of individual personality types since its development in the 1940s. His theory is rooted in the idea that individuals are born with inherent preferences on how they use their mind, and the MBTI is a questionnaire based on this theory that measures personality types using four dichotomous categories: extroversion-introversion (E-I), sensing-intuition (S-N), thinking-feeling (T-F), and judgingperception (J-P). These indicators are meant to analyze an "individual's preferred method of interpreting and using information and how they relate to the outside world"1.

The relationships within each category are described as follows: the E-I dichotomy explores whether individuals tend to gather energy from within themselves via personal reflection (I), or from social interaction from the outside world (E). The S-N category differentiates between individuals who prefer to take in information based on facts $(\mathrm{S})$ versus possibilities (N). T-F describes how people tend to make decisions based on logic and objective findings $(\mathrm{T})$, or subjective and personal reasons (F). Finally, the J-P dimension is about whether individuals prefer to deal with the outside world in a sharply organized and planned fashion (J), or a more spontaneous, flexible approach $(\mathrm{P})$.

Based on these categories, individuals receive 1 of 16 possible unique personality designations in the form of a four-letter profile. This tool has been used in the past to better understand leadership building, team development, and interpersonal communications strategies. ${ }^{2}$ Over the years, this survey has been applied to examine patterns in career choice and tendencies in many fields of work, including medical specialties.

Studies have suggested that personality types can influence medical specialty selection, applicant rankings, as well as certain aspects of resident education. As early as the 1950s, Isabel Meyers-one of the founders of the toolauthored a study that showed that physicians were more likely to identify with the J personality category. ${ }^{3}$ Later studies supported these findings in medicine, and further explored these results in various specialties. For example, E and $\mathrm{T}$ personality types have consistently been more likely to pursue a surgical specialty than their I and $\mathrm{F}$ counterparts. ${ }^{4}$ In a study of orthopedic residency applicants, it was found that when ranking applicants for the match, faculty were more likely to rank applicants with similar personalities to themselves. ${ }^{5}$ In the examination of general surgery, it was found that differing personality types have effects on teacherlearner interaction during clerkships. ${ }^{6}$ Additional studies have evaluated MBTI types in psychiatry, pediatrics, anesthesiology, and physical medicine and rehabilitation. ${ }^{7-11}$

Ophthalmology represents a unique specialty within medicine for its combination of outpatient clinical practice and highly specialized surgical procedures. The closest existing study evaluated otolaryngology residency applicants, noting an increased prevalence of ISTJ, ESTJ, and ENTJ personality types within the specialty. ${ }^{1}$ With this in mind, we used the MBTI to analyze personality type among current ophthalmology residents and recent graduates. We aimed to evaluate the prevalence of specific personality types, and whether these varied by level of training, training program, or fellowship selection.

\section{Participants and Methods}

After obtaining institutional review board approval from Howard University Hospital, an electronic version of the MBTI questionnaire, form M, was sent to ophthalmology residents of five participating programs. Participants included current residents (PGY2-PGY4) and recent graduates ("attendings") of Howard University, Georgetown, George Washington, University of Texas Medical Branch at Galveston (UTMB), and Kresge Eye Institute. The survey consisted of 93 questions, with an additional four questions inquiring about home program, postgraduate training level, subspecialty interest, and work environment (if applicable). The anonymous responses of the surveys were automatically scored on google forms, and the results were analyzed by using StatView statistical analysis.

\section{Results}

A total of 66 of 138 individuals were responded to the survey request (response rate $=48 \%$ ), and all five training programs were represented (-Fig. 1). This included 47 out of 69 current residents as well as 19 out of 69 graduates of the past 3 years (response rate of 68 and $28 \%$, respectively). The total response rate of $48 \%$ is broken down by program as follows: Howard (77.8\%), George Washington (70.8), UTMB 


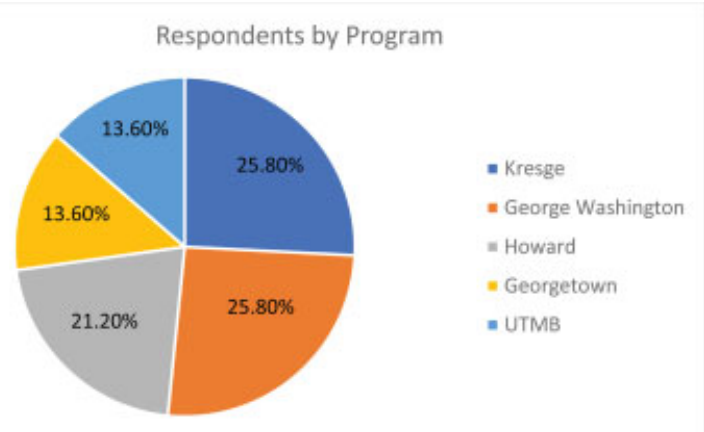

Fig. 1 Distribution of survey respondents by residency program.

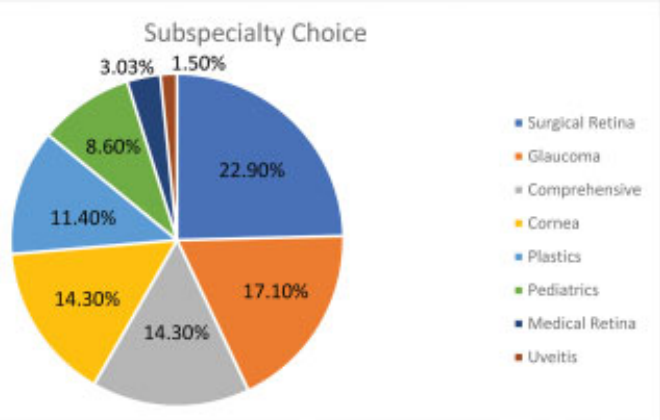

Fig. 2 Distribution of sample respondents by subspecialty choice.

(50\%), Kresge (40.5\%), and Georgetown (25\%). However, 14 out of 16 of the MBTI personality types were represented in the poll results. Of the recent graduates surveyed, subspecialty representation included surgical retina, glaucoma, comprehensive practice, cornea, oculoplastics, pediatrics, medical retina, and uveitis (-Fig. 2).

The extraversion (E)/introversion (I) results significantly favored the extraversion category at 62 versus $38 \%$ for introversion $(p=0.049)$. Sensing $(\mathrm{S})$ versus intuition $(\mathrm{N})$ were not significantly different at 56 versus $44 \%$ $(p=0.325)$. Ophthalmology trainees were more likely to be categorized as thinking $(\mathrm{T})$ instead of feeling $(\mathrm{F})$ at 64 versus
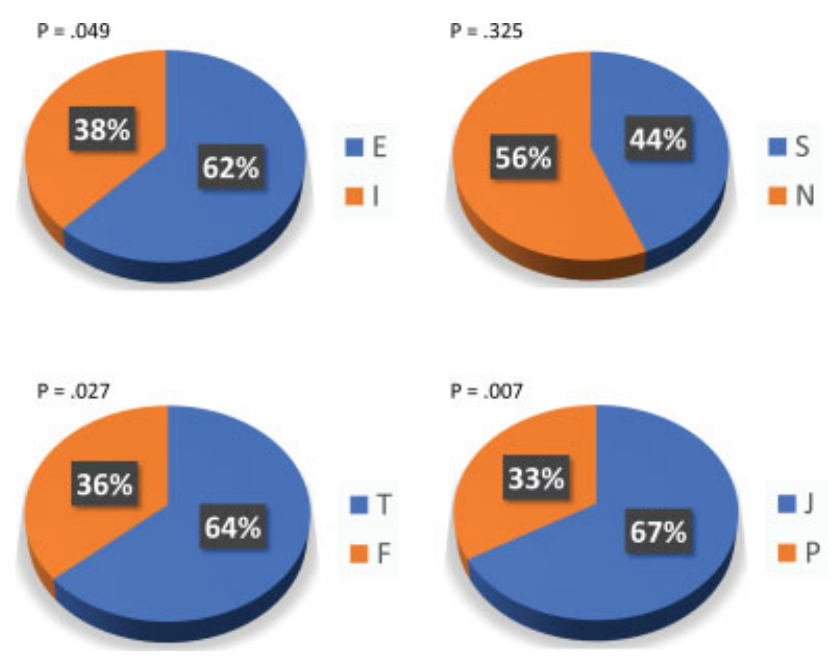

Fig. 3 Distribution of all respondents in the four dichotomous categories.

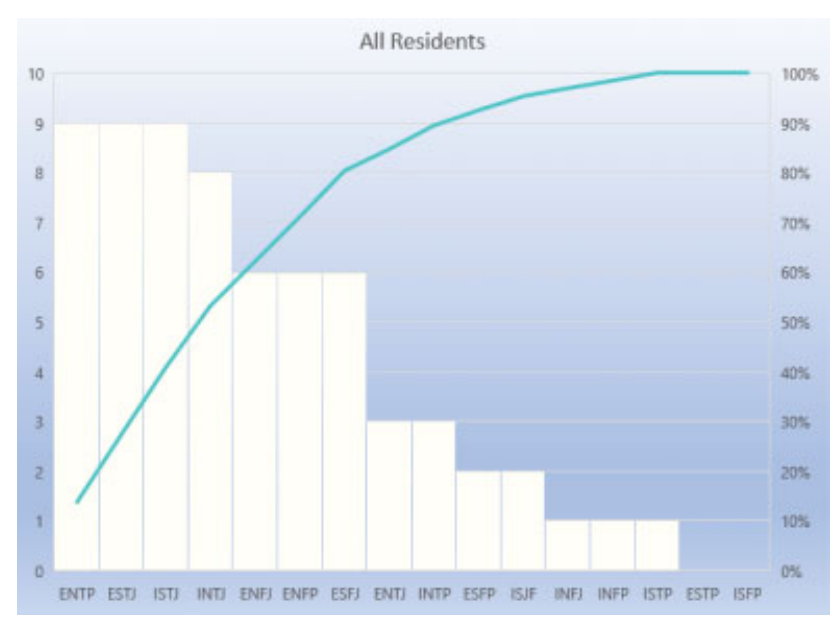

Fig. 4 Distribution of all respondents among the 16 unique personality types.

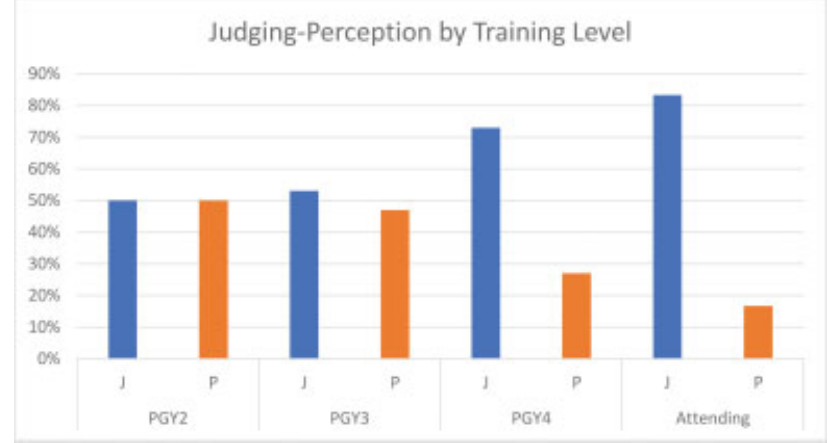

Fig. 5 Judging-perception dichotomy examined by training level.

$36 \%(p=0.027)$, and judging $(\mathrm{J})$ instead of perceiving $(\mathrm{P})$ at 67 versus $33 \%(p=0.007)$. These results are summarized in the graphical depictions of $\mathbf{- F i g . ~} 3$.

The most common unique MBTI personality types among residents were ENTP (13.6\%), ESTJ (13.6\%), ISTJ (13.6\%), and INTJ (12.1\%). Conversely, the least common were ISFP/ESTP (0\%) and INFJ/INFP/ISTP (1.5\%) as shown in - Fig. 4.

The judging-perceiving dichotomy was particularly unique in that it seemed to change by level of training (-Fig. 5). At the PGY2 level, the J:P percentage ratio was 50:50. For PGY3, it was 53:47. For PGY4, it was 73:27. For graduates, it was 83:17.

\section{Discussion}

To our knowledge, there is no published literature specifically examining the personality types of ophthalmology residents. Some specialties that have been examined using MBTI personality types include general surgery, orthopedic surgery, psychiatry, physical medicine and rehabilitation (PM\&R), anesthesiology, and pediatrics. ${ }^{5-11}$ The closest existing comparison, however, is a study of medical students applying to otolaryngology by Zardouz et al in $2010 .{ }^{1}$ Akin to ENT and previously under the same training, ophthalmology is a surgical specialty that attracts competitive applicants 
who are drawn to solving well-defined problems with tangible results. It is relatively unique in medicine for its outpatient nature with a focus on surgical procedures and ever evolving technologies. As such, our study provides valuable insight into potential personality types in this field.

In terms of unique personality types, it was found that ESTJ, ENTJ, and ISTJ were the three most common MBTI indices represented among ophthalmology residents. These three personalities each occurred a prevalence of $13.6 \%$ in our study, which is more frequent than their prevalence in the general population, which are estimated at 11,6 , and $10 \%$, respectively. ${ }^{12}$ This could be important in understanding why certain individuals are drawn toward ophthalmology as a specialty, or perhaps the fostering of these personality trends once accepted in the specialty. While this is useful information, it would be valuable to take this one step further and additionally examine prevalence among ophthalmology applicants, as Zardouz et al did with ENT. If similar prevalence rates were found in ophthalmology applicants compared with residents, then this might suggest that personality types influence interest in specialty. If rates were found to be different, however, then this might suggest potential selection association based on personality type. It is certainly worth noting that two of the three most prevalent personality types in our study were the same as in the study on ENT applicants: ESTJ and ISTJ. ${ }^{1}$ Given the similarities between the specialties, this may suggest that these common personalities are attracted to these fields rather than being influenced by it.

The three most common personalities in our study are described in the MBTI handbook as follows: "ESTJ personality types are analytical, goal-oriented, decisive and organized." "ISTJ personality types are sensible and reliable and pay attention to detail." "ENTJ personality types are driven, organized, and decisive natural leaders." ${ }^{2}$ When examining each dichotomous category, ophthalmology trainees were statistically more likely to be extroverted $(\mathrm{E})$, thinking $(\mathrm{T})$, and judging $(J)$, with no difference between sensing/intuition $(\mathrm{S} / \mathrm{N})$. This builds on a similar finding from Stilwell et al, where it was found that individuals with $\mathrm{E}$ and $\mathrm{T}$ personalities were more likely to seek out surgical specialties. ${ }^{4}$ Their study did not, however, show an increased likelihood of the J personality as we did. This poses the question of whether these findings are specifically unique to ophthalmology or perhaps a product of ophthalmology as a surgical specialty.

With regards to differences by level of training, there was a notable change in the J:P ratio per PGY level. The prevalence of the judging profile increased steadily with each year of residency and into the graduate category, starting with a J:P ratio of 50:50 in the PGY2 level, eventually leading to a ratio of 83:17 in the graduates. This suggests that the judging category, in particular, may be influenced over the course of one's training and experience. This particular finding is consistent with the results seen in Sliwa et $\mathrm{al}^{9}$ as well as Contessa et $\mathrm{al}^{11}{ }^{11}$ in their research on PM\&R and surgery residents, respectively. Both studies found the "graduates" to be significantly more judging than the "residents." They did not, however, separate the "residents" group into multiple groups by training year. In fact, to our knowledge, our study is the only one that examines this stepwise change in personality from year to year. This finding could have been further strengthened by dividing the graduate category into years of practice. Further studies could investigate this trend further. Additionally, to best evaluate if individual personalities change as training continues, a longitudinal study could be conducted.

Among training programs and subspecialty choices, variations in personality were found; however, statistical analysis was limited by the small sample size in this population, and in order for statistical significance to be examined, a larger study should be conducted. Further limitations of this study included selection bias and response bias. It must be acknowledged that the response rates varied between training programs and between graduates and residents as well.

Our study highlights the fact that ophthalmology residents were more likely to identify with certain personality types and traits as measured by the MBTI inventory. A more widely administered survey would be helpful to verify these findings, as well as to explore other associations between personality types and residency training. Our study comes at a time when the United States Medical Licensing Examination step 1 will be transitioning to a pass/fail system, potentially minimizing the influence of a historically important factor in a competitive application process. If future studies yield similar findings to our own, personality inventories may provide applicants and programs with a supplemental tool worthy of consideration.

\section{Conclusion}

Certain personality types are more common among ophthalmology residents in our cohort from five different training programs. Personality types may vary by subspecialty choice and training program. Furthermore, it is possible that individual types change over the course of residency training and career. Understanding that these findings exist can be used as a baseline for future research in terms of potential predictors for applicants, of resident knowledge base, and for personality changes over the course of one's training. Further research should aim to reach a larger, more comprehensive sample of trainees to account for selection and response biases.

\section{Funding \\ None. \\ Conflict of Interest \\ None declared.}

\section{References}

1 Zardouz S, German MA, Wu EC, Djalilian HR. Personality types of otolaryngology resident applicants as described by the MyersBriggs Type Indicator. Otolaryngol Head Neck Surg 2011;144(05): 714-718

2 CPP, Inc. Myers Briggs Type Indicator assessment. Accessed 2007 at: www.cpp.com/products/mbti/index.aspx

3 Myers IB, Davis JA. Relation of medical students' psychological type to their specialties twelve years later. Princeton, NJ: Educational Testing Service; 1965 
4 Stilwell NA, Wallick MM, Thal SE, Burleson JA. Myers-Briggs type and medical specialty choice: a new look at an old question. Teach Learn Med 2000;12(01):14-20

5 Quintero AJ, Segal LS, King TS, Black KP. The personal interview: assessing the potential for personality similarity to bias the selection of orthopaedic residents. Acad Med 2009;84(10):1364-1372

6 Bell MA, Wales P, Torbeck L, et al. Do personality differences between teachers and learners impact students evaluation of surgical clerkship? J Surg Educ 2011. Doi: 10.1016/j.jsurg.2011.01.003

7 Yang C, Richard G, Durkin M. The association between MyersBriggs Type Indicator and Psychiatry as the specialty choice. Int J Med Educ 2016;7:48-51

8 Schell RM, Dilorenzo AN, Li HF, Fragneto RY, Bowe EA, Hessel EA II. Anesthesiology resident personality type correlates with faculty assessment of resident performance. J Clin Anesth 2012;24(07): 566-572

9 Sliwa JA, Shade-Zeldow Y. Physician personality types in physical medicine and rehabilitation as measured by the Myers-Briggs Type Indicator. Am J Phys Med Rehabil 1994;73(05):308-312

10 Lacorte MA, Risucci DA. Personality, clinical performance and knowledge in paediatric residents. Med Educ 1993;27(02): 165-169

11 Contessa J, Suarez L, Kyriakides T, Nadzam G. The influence of surgeon personality factors on risk tolerance: a pilot study. J Surg Educ 2013;70(06):806-812

12 Macdaid GP, McCaulley M, Kainz R. Myers-Briggs Type Indicator: Atlas of Type Tables. Palo Alto, CA: Consulting Psychologists Press; 1986 